\title{
Forecasting for Air Material Consumption Based on Winters Exponential Smoothing Model
}

\author{
Yanming Yang*, Wenxiu Wang and Chaoran Guo \\ Naval Aeronautical and Astronautical University Qingdao Campus; Qingdao 266041; China \\ *yymqd@126.com
}

Keywords: Winters Model, Seasonal Forecasting, Air Material Consumption.

Abstract. It is indispensable to scientifically predict the consumption of air material spare parts and to make scientific decisions on aviation equipment maintenance resources and make full use of existing resources to improve maintenance capability. In the process of aviation equipment maintenance and support, the consumption of air material spare parts tends to show a seasonal change. In this paper, the mathematic model and calculation method of Winters exponential smoothing are discussed. The application of Winters model in forecasting the consumption of air material spare parts is analyzed by examples. And the forecasting results were analyzed and compared. At last, the results show that the Winters model is feasible and effective for the prediction of air material spare parts consumption.

\section{Introduction}

The air material spare parts are essential material basis for aviation equipment maintenance engineering. With the development of aviation equipment, more and more complex equipment, maintenance of spare parts required for the variety and quantity are more and more spare parts financing, supply and storage process is also more complex. Spare parts are often seasonal changes in consumption, this seasonal changes in the aviation parts of the financing raised the challenge. Winters exponential smoothing method is an effective method to solve this kind of problem. In this paper, we use Winters exponential smoothing method to forecast the consumption of spare parts.

\section{Winters Exponential Smoothing Model}

Winters model employs a level component, a trend component, and a seasonal component at each period. It uses three weights, or smoothing parameters, to update the components at each period. Initial values for the level and trend components are obtained from a linear regression on time. Initial values for the seasonal component are obtained from a dummy-variable regression using detrended data. Suppose we have a sequence of observations $\left\{x_{t}\right\}$, beginning at time $t=0$ with a cycle of seasonal change of length $p$. The following describes the basic principles of Winters model with multiplicative seasonality.

Smoothing Equations. The three basic formulas of Winters model are:

$$
\begin{aligned}
& L_{t}=\alpha \frac{Y_{t}}{I_{t-p}}+(1-\alpha)\left(S_{t-1}+T_{t-1}\right) \\
& T_{t}=\gamma\left(L_{t}-L_{t-1}\right)+(1-\gamma) T_{t-1} \\
& S_{t}=\delta \frac{Y_{t}}{L_{t}}+(1-\delta) S_{t-p}
\end{aligned}
$$

Where $\alpha$ is the data smoothing factor, $0<\alpha<1 ; \gamma$ is the trend smoothing factor, $0<\gamma<1$; $\delta$ is the seasonal change smoothing factor, $0<\delta<1$; $p$ is the seasonal period; $L_{t}$ is the level at time $t$; $T_{t}$ is the trend at time $t ; S_{t}$ is the seasonal component at time $t ; Y_{t}$ is the data value at time $t$. 
Prediction Equation. The prediction formula of Winters model:

$\hat{Y}_{t}=\left(L_{t}+T_{t-1}\right) S_{t-p}$

Where $\hat{Y}_{t}$ is the fitted value, or one-period-ahead forecast, at time $t$.

Initial Values of Model Parameters. The general formula for the initial trend estimate $T_{0}$ is:

$$
T_{0}=\frac{1}{p}\left(\frac{x_{p+1}-x_{1}}{p}+\frac{x_{p+2}-x_{2}}{p}+\ldots+\frac{x_{p+p}-x_{p}}{p}\right)
$$

Setting the initial estimates for the seasonal indices $S_{0}$ for $i=1,2, \ldots, p$ is a bit more involved. If $N$ is the number of complete cycles present in your data, then:

$$
S_{i}=\frac{1}{N} \sum_{j=1}^{N} \frac{x_{p(j-1)+i}}{A_{j}} \quad, \quad i=1,2, \ldots, p
$$

Where

$$
A_{j}=\frac{\sum_{i=1}^{p} x_{p(j-1)+i}}{p}, j=1,2, \ldots, N
$$

Note that $A_{0}$ is the average value of $x$ in the $j$ th cycle of your data.

\section{Application Example Analysis}

Problem Description. In the period from 2012 to 2016, the consumption of spare parts for certain aeronautical materials is shown in Table 1. Taking into account the seasonal influence of the air material consumption, the Winters model is used to predict the consumption of the spare parts in 4 quarters in 2017.

Table 1. Consumption of air materials spare parts in 2012 2016

\begin{tabular}{|c|c|c|c|c|}
\hline Year & First Quarter & Second Quarter & Third Quarter & Fourth Quarter \\
\hline 2012 & 137 & 186 & 274 & 175 \\
\hline 2013 & 142 & 198 & 265 & 183 \\
\hline 2014 & 131 & 193 & 247 & 169 \\
\hline 2015 & 157 & 200 & 283 & 194 \\
\hline 2016 & 149 & 214 & 276 & 185 \\
\hline
\end{tabular}

By plotting the time series, as shown in Figure 1, it can be seen that the historical data of the consumption of air material spare parts exhibits obvious seasonal (periodic) characteristics. Therefore, the Winters model can be used to predict.

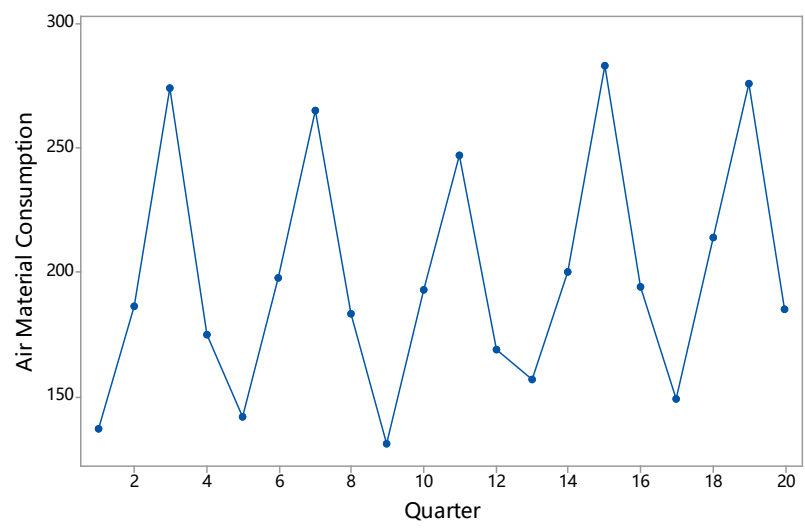

Figure 1. Time series plot of air material consumption 
Predictive Results. Using the time series analysis function of Minitab software (Winters method) to obtain the following prediction results (as shown in Table 2) and forecast (as shown in Figure 2).

Table 2. The forecast of air materials consumption in each quarter of 2017

\begin{tabular}{|l|c|c|c|}
\hline \multirow{2}{*}{ Time } & \multirow{2}{*}{ Forecast } & 95\% Normal Confidence Interval \\
\cline { 3 - 4 } & & Lower & Upper \\
\hline The First Quarter of 2017 & 153.957 & 87.539 & 220.374 \\
\hline The Second Quarter of 2017 & 210.244 & 142.786 & 277.703 \\
\hline The Third Quarter of 2017 & 281.963 & 119.392 & 350.582 \\
\hline The Fourth Quarter of 2017 & 189.285 & 119.39 & 259.178 \\
\hline
\end{tabular}

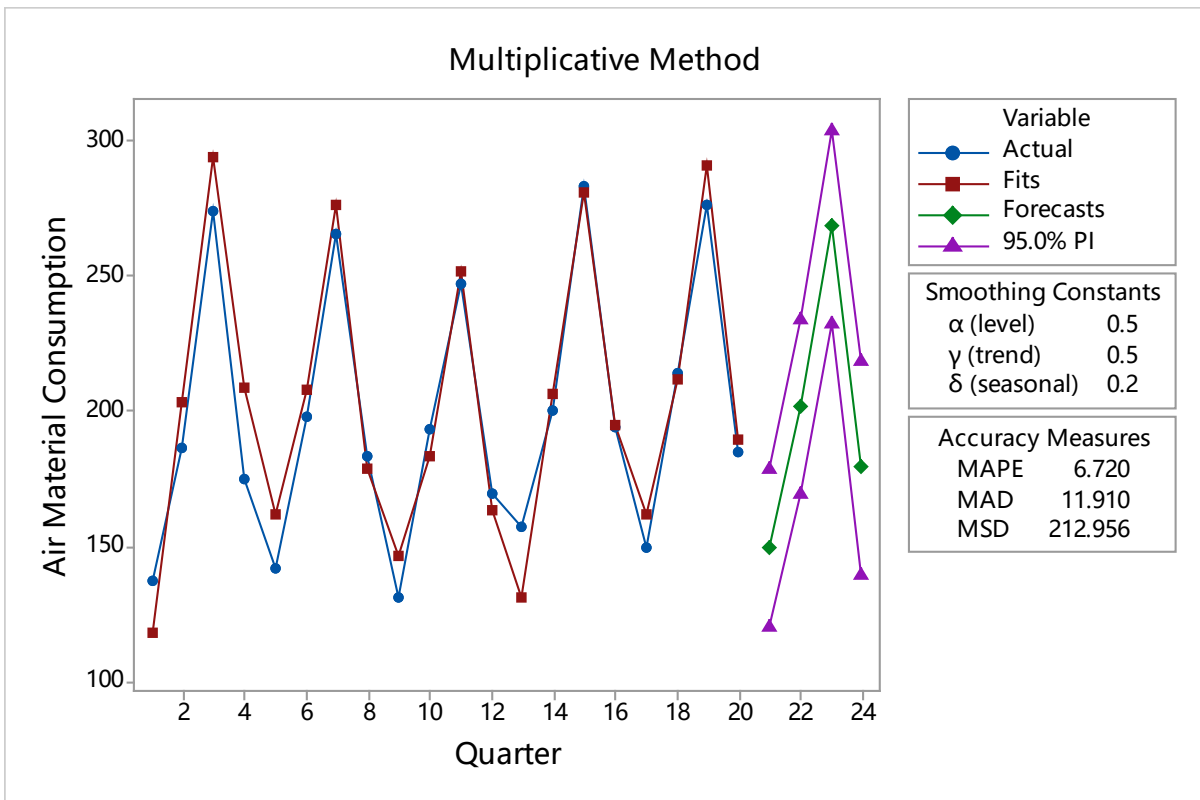

Figure 2. Winters method plot for air material consumption after adjusting the smoothing parameter

Residual Plots Analysis. Use to examine the goodness of model fit in regression and ANOVA. Examining residual plots helps you determine if the ordinary least squares assumptions are being met. If these assumptions are satisfied, then ordinary least squares regression will produce unbiased coefficient estimates with the minimum variance. Minitab provides the following residual plots (as shown in Figure 3):
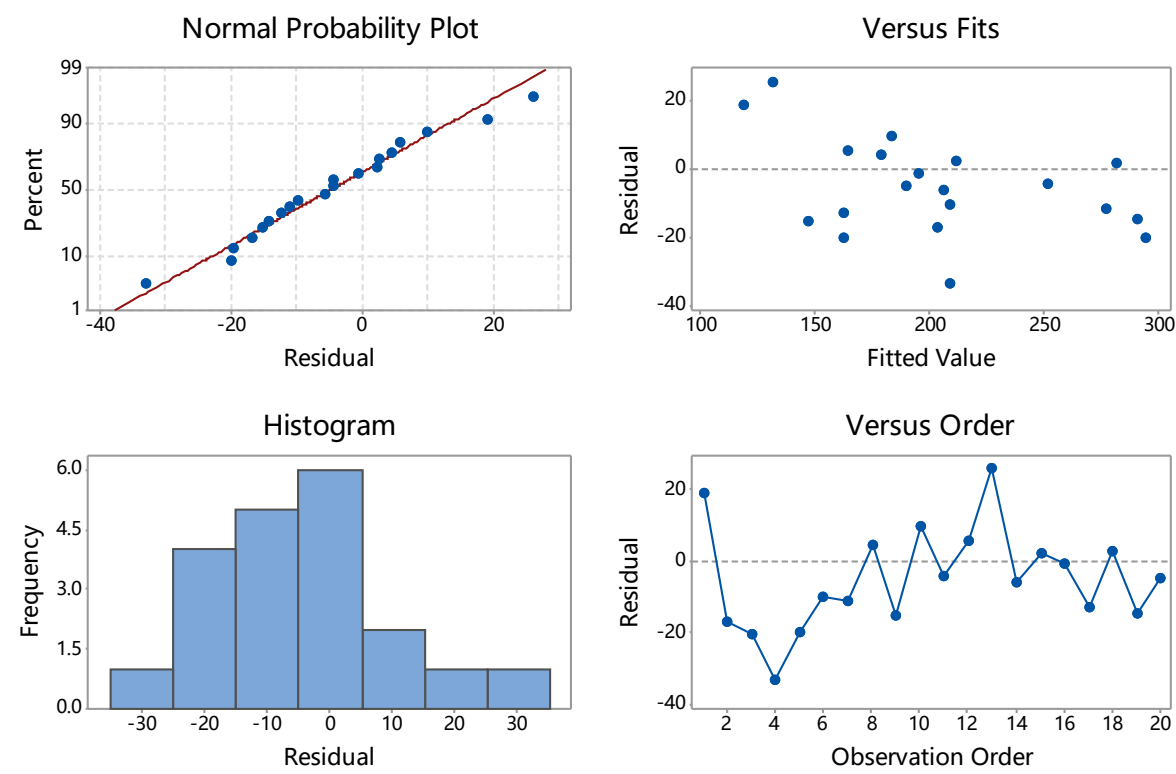

Figure 3. Residual plots for air material consumption after adjusting the smoothing parameter 
Normal Probability Plot of residuals. The points in this plot should generally form a straight line if the residuals are normally distributed. If the points on the plot depart from a straight line, the normality assumption may be invalid.

Histogram of the Residuals. An exploratory tool to show general characteristics of the residuals including typical values, spread, and shape. A long tail on one side may indicate a skewed distribution. If one or two bars are far from the others, those points may be outliers.

Residuals Versus Fitted Values. This plot should show a random pattern of residuals on both sides of 0 . If a point lies far from the majority of points, it may be an outlier. There should not be any recognizable patterns in the residual plot. For instance, if the spread of residual values tend to increase as the fitted values increase, then this may violate the constant variance assumption.

Residuals Versus Order of Data. This is a plot of all residuals in the order that the data was collected and can be used to find non-random error, especially of time-related effects. This plot helps you to check the assumption that the residuals are uncorrelated with each other

Residuals versus predictors. This is a plot of the residuals versus a predictor. This plot should show a random pattern of residuals on both sides of 0 . Non-random patterns, such as the example to the right, may violate the assumption that predictor variables are unrelated to the residuals. You may have used an incorrect functional form to model the curvature.

\section{Conclusions}

The Winters exponential smoothing is used when the data exhibits both trend and seasonality. The two main Winters models are Additive model for time series exhibiting additive seasonality and Multiplicative model for time series exhibiting Multiplicative seasonality. The application example shows that the Winters method can make full use of historical data, and can accurately forecast the consumption of air material by depicting the periodic fluctuation. Moreover, the Winters method is simple and effective, and it can predict the consumption of spare parts in one cycle at a time. It is an effective short-term and medium-term prediction method. At the same time, the Winters method proposed in this paper not only applies to the prediction of air material spare parts consumption, but also to other equipment indexes or parameters with time series characteristics, such as equipment failure rate, flight safety accident rate and aviation equipment readiness rate, which provides a scientific method and means for equipment support forecast.

\section{References}

[1] MLAGoodwin, Paul. The Holt-Winters Approach to Exponential Smoothing: 50 Years Old and Going Strong. Foresight the International Journal of Applied Forecasting 19(2010):30-33.

[2] Kalekar, Prajakta S. Time series Forecasting using Holt-Winters Exponential Smoothing. Kanwal Rekhi School of Information Technology (2004).

[3] Winters, P. R. (April 1960). Forecasting Sales by Exponentially Weighted Moving Averages. Management Science. 6 (3): 324-342.

[4] Charles C. Holt. Forecasting Trends and Seasonal by Exponentially Weighted Averages. International Journal of Forecasting. 20 (1): 5-10.

[5] Hyndman, Rob, et al. Forecasting with Exponential Smoothing. Springer 26.1(2008):204-205.

[6] Osman, Ahmad Farid, and M. L. King. A new approach to forecasting based on exponential smoothing with independent regressors. Monash Econometrics \& Business Statistics Working Papers (2015).

[7] Information on https://en.wikipedia.org/wiki/Exponential_smoothing. 